\title{
Spontaneous retroperitoneal and subcapsular liver hematoma. The diagnostic contribution of CT, US and CEUS. Case report.
}

\author{
Radu Badea ${ }^{1}$, Liliana Chiorean ${ }^{2}$, Calin Mitre ${ }^{3}$, Carolina Botar-Jid ${ }^{4}$, Cosmin Caraiani $^{2}$
}

${ }^{1}$ Ultrasonography Department, $3^{\text {rd }}$ Medical Clinic, “Octavian Fodor” Regional Institute of Hepatology and Gastroenterology, ’Iuliu Hațieganu“ University of Medicine and Pharmacy, Cluj-Napoca, Romania

${ }^{2}$ Radiology Department, $3^{\text {rd }}$ Medical Clinic, “Octavian Fodor" Regional Institute of Hepatology and Gastroenterology, Cluj-Napoca, Romania

${ }^{3}$ Intensive Care Department, $3^{\text {rd }}$ Surgery Clinic, "Octavian Fodor" Regional Institute of Hepatology and Gastroenterology, ’Iuliu Hațieganu“ University of Medicine and Pharmacy Cluj-Napoca, Romania

${ }^{4}$ Radiology Department, Emergency County Hospital Cluj Napoca, ”Iuliu Hațieganu“ University of Medicine and Pharmacy Cluj-Napoca, Romania

\section{Abstract}

We present the case of a patient undergoing long-time treatment with oral anticoagulants for a severe vascular pathology who had a major subcapsular liver bleeding as well as retroperitoneal bleeding. The purpose of the paper is to present the specific characteristics of this case, to review the literature that concentrates on spontaneous bleedings and to show the role of contrast-enhanced ultrasonography as a useful, non invasive imaging method in excluding active hemorrhage and its value in the non-surgical management of the case.

Keywords: subcapsular liver hematoma, oral anticoagulants, contrast-enhanced ultrasonography

\section{Introduction}

Abdominal hemorrhages have numerous causes among which: trauma, coagulopathies, post surgical conditions or interventional medical procedures performed either for diagnosis or therapeutic purposes. Medical literature recognizes the term "spontaneous abdominal hemorrhage" as the presence of intra-abdominal blood due to a non-traumatic, non-iatrogenic cause [1]. Abdominal bleeding due to anticoagulant treatment or hem-

Received 23.01.2013 Accepted 16.02.2013

Med Ultrason

2013, Vol. 15, No 2, 157-160

Corresponding author: Prof Radu Badea MD, PhD

Department of Ultrasonography,

$3^{\text {rd }}$ Medical Clinic, Gastroenterology and

Hepatology Institute,

"Iuliu Hațieganu" University of Medicine and Pharmacy,

19-21 Croitorilor str

400494 Cluj Napoca, Romania

Email: rbadea@umfcluj.ro orrhagic diathesis (liver failure, hemophilia, idiopathic thrombocytopenic purpura, systemic lupus erythematosus) develops in various places simultaneously, arising especially in the abdominal muscles (rectus abdominis or psoas muscle). The abdominal organs are not involved as often, the hemorrhage in these situations developing in the perirenal space or in the intestinal walls (mainly jejunum) $[2,3]$. In patients undergoing chronic oral anticoagulant treatment gastric hematomas [4] and a few rare cases of bleeding within the lumen or the wall of the gall bladder have been reported [5]. Another possible bleeding site is the retroperitoneum, which is a rare but lifethreatening situation [6]. There have been reported rare cases of subcapsular liver hematomas in patients with Wegener Granulomatosis [7] and post Imatinib administration for treatment of metastatic GIST [8].

Imaging explorations have a decisive role in the detection and characterization of hematomas [9]. The use of contrast enhanced ultrasonography (CEUS) as a diagnosis imaging technique on a larger scale may contribute to 
the change of the therapeutic management in abdominal hemorrhages, from an invasive approach towards a nonsurgical one, an aspect which has presented an increased interest in the last years [10].

\section{Case report}

A 68 year old patient presented in the emergency department for intense, diffuse abdominal pain, abdominal distension, epistaxis, and bloody stools. The onset of the symptoms had been a few days before the presentation and became more and more intense, with the peak of intensity in the day before presentation. The patient's history included a bilateral aortofemoral bypass intervention in 1994 for lower limbs arteriopathy obliterans. Since then the patient had received chronic treatment with oral anticoagulants (Sintrom $14 \mathrm{mg}$ and Acetylsalicylic Acid 40 $\mathrm{mg}$ /day). The patient did not report any trauma or infectious episodes in recent history and did not use alcohol or any other drugs. The lab investigations performed in the emergency department revealed severe anemia $(\mathrm{Hb}=7 \mathrm{mg} /$ $\mathrm{dl} ; \mathrm{Ht}=27 \%$ ) as well as important alterations of the coagulation status with INR $>9$ (International Normalized Ratio).

The abdominal computed tomography (CT) examination performed at presentation revealed the presence of a hypodense fluid collection surrounding the left lobe of the liver (fig 1a), as well as fluid in the retroperitoneal space (fig 1b). The patient was admitted into an intensive care facility for further investigations and therapy. An ultrasound (US) examination was performed (fig 2), followed by a CEUS exam (fig 3) which confirmed the presence of a large subcapsular liver fluid collection and a second collection, located on the inferior surface of the liver communicating with the first collection. Both fluid collections were well circumscribed and presented a heterogeneous content, with membrane-like echoes inside, an aspect typical for the presence of blood clots. The retroperitoneal collection was also detected. The diagnosis of subcapsular liver hematoma (around the left lobe of the liver) and retroperitoneal hemorrhage was established, the absence of micro-bubbles inside the collection being highly suggestive for inactive hemorrhage.

Based on this diagnosis, plasma and blood were transfused, resulting in a favorable evolution of the patient (within a week from admission the anemia was resolved, the INR reached normal values and there was remission of the clinical complaints). The follow up US examination revealed the tendency of the subcapsular liver hematoma towards organization, while the CT exam showed a reduction of the retroperitoneal fluid collection. The patient was released with an improved status without undergoing surgery.

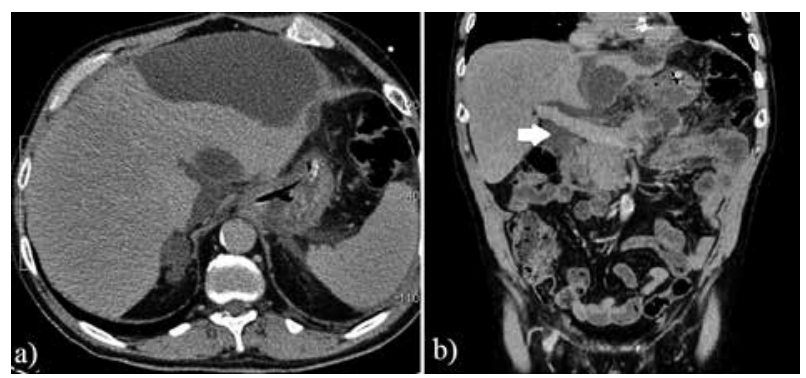

Fig 1. CT examination. a) Axial view, arterial phase, showing a hypodense fluid collection around the left lobe of the liver; b) 3D reconstruction, coronal view, arterial phase - subcapsular fluid collection surrounding the left liver lobe and fluid collection in the retroperitoneal space (arrow).
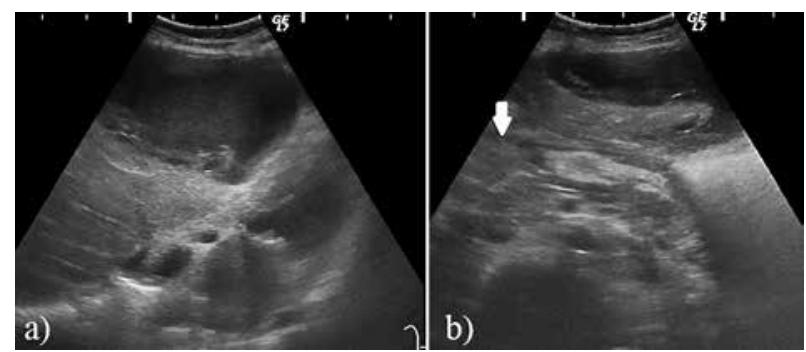

Fig 2. Bidimensional ultrasonographic examination, transverse view: a) Subcapsular liver collection - there is an echoic sedimentation inside the collection, suggestive for blood content; b) Subcapsular liver collection and retroperitoneal collection (arrow).

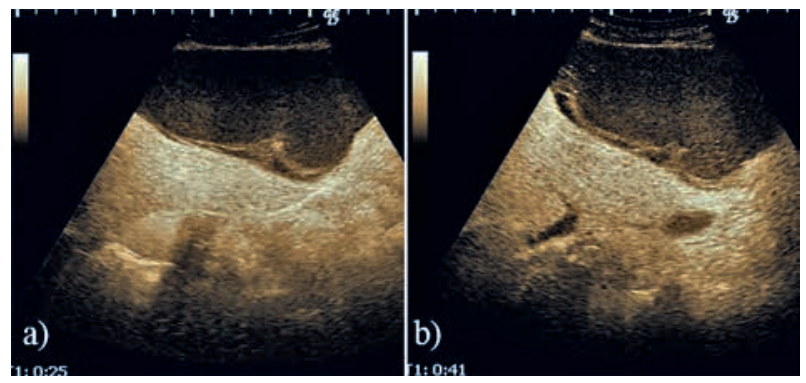

Fig 3. CEUS examination. a) Arterial phase - there are no micro-bubbles inside the collection; b) Portal phase - the aspect identified during the arterial phase is also detected during the portal phase.

\section{Discussions}

Subcapsular liver hematomas represent an accumulation of blood between the capsule of Glisson and the liver parenchyma. They are frequently located around the right lobe (about $75 \%$ of cases) and the rupture of the hematoma into the peritoneum is associated with a high 
mortality rate [11]. The development mechanism of subcapsular liver hematomas, as well as their self-limiting character is conditioned by the anatomy of the liver capsule. This is made of two layers: the first is the visceral layer (capsule of Glisson) in close contact with the liver parenchyma, and the second is actually a reflection of the peritoneum sustaining the liver in contact with the diaphragm (hepatodiaphragmatic ligament) [12]. Since the capsule of Glisson is fibrous and has no elasticity, the shape of the hematoma is convex towards the parenchy$\mathrm{ma}$, an aspect that helps to distinguish a hematoma from other perihepatic not subcapsular collections, in which the shape of the liver is not modified by compression. The symptoms of liver hematomas are not specific, the laboratory studies reveal anemia and coagulation alterations and the final diagnosis is established through imaging techniques (ultrasonography and CT) $[1,13]$.

The necessity of an early and accurate diagnosis in subcapsular liver hemorrhages derives from the modern trend of treating them conservatively, especially when the patient is hemodynamically stable [10]. Therefore a lot is expected from imaging procedures. On the CT examination a subcapsular liver hematoma typically looks like a lenticular, ellipsoid, perihepatic collection with a density that on the non-enhanced exam depends on the "age" of the hematoma. Acute hematomas are typically hyperdense (40-60 HU), due to the high protein content. The density decreases in time due to the progressive lysis of hemoglobin becoming hypodense in the chronic phase [14].

The ultrasonographic appearance of hematomas varies depending on their localization and "age": a recent hematoma has a fluid-like aspect, transonic and, well circumscribed by the capsule of the organ; there can be sedimentation inside or even intense echoes generated by the blood clots. In time, a few days after formation, the hematoma becomes echoic by condensation and theaccumulation of clots [15]. Therefore, the main diagnosis criteria is represented by changes in the aspect of the collection from one day to another, the resorption of the collection being influenced by the context in which the hemorrhage has occurred, comorbidities, continuous or ceased bleeding and the volume of the bleeding.

Regarding the evolution of the hematoma, CEUS is extremely useful as a diagnosis and follow up imaging method. Once the blood collection is detected, CEUS helps to better evaluation and measurement [16]. An enhancement of parenchyma that surrounds the collection is registered during the arterial phase and the aspect persists during the venous and delayed phases. The method is very sensitive in detecting active bleeding by visualizing the echoes inside the collection as well as in detecting possible vessels malformations that can cause the bleed- ing [17]. CEUS is a "real-time", non-invasive, bedside investigation that does not require radiation exposure and is an adequate choice for the evaluation of abdominal solid organs in patients that have contraindications for CT contrast media administration or are hemodynamically unstable [18].

There is quite an abundance of literature regarding spontaneous hemorrhages and the value of imaging for diagnosis. The specific features of this case are represented by the fact that a spontaneous, massive subcapsular liver hematoma in a patient with an excessive intake of anticoagulants is rare and the association with spontan retroperitoneal bleeding is even rarer. We also wanted to highlight the use that CEUS had to confirm that the bleeding has stopped. The diagnosis algorithm that was used, based on the imaging data, led to a non-invasive therapeutic approach. The complexity of the differential diagnosis must also be underlined, as the magnitude of the hemorrhage, both subcapsular and retroperitoneal, raised significant differential diagnosis problems, both on the CT and US images. Therefore, in this case, the correlation of the imaging data with the patient's history was very important.

Conclusions: Imaging investigations allow the detection of internal hemorrhages and help establish the organ which is involved, the site, the extent and the evolutive features of the bleeding. CEUS is useful to consolidate the clinical diagnosis of a stopped hemorrhage. The hemorrhagic character of a fluid collection is demonstrated by observing the changes of the US aspect in dynamic examination. When there is hemodynamic stability the imaging data are sufficient for the diagnosis in emergency situations, making other invasive procedures unnecessary.

\section{References}

1. Furlan A, Fakhran S, Federle MP. Spontaneous abdominal hemorrhage: causes, CT findings and clinical implications. AJR Am J Roentgenol 2009; 193: 1077-1087.

2. Birla RP, Mahawar KK, Saw EY, Tabaqchali MA, Woolfall P. Spontaneous intramural jejunal haematoma: a case report. Cases J 2008; 1: 389.

3. Zissin R, Ellis M, Gayer G. The CT findings of abdominal anticoagulant-related hematomas. Semin Ultrasound CT MR 2006; 27: 117-125.

4. Mânzat Săplăcan RM, Cătinean A, Manole S, Vălean SD, Chira RI, Mircea PA. Posttraumatic gastric wall hematoma in a patient under anticoagulant therapy. Case report and literature review. Med Ultrason 2011; 13: 165-170.

5. Pilling DW. Haematoma of the wall of the gall-bladder. $\mathrm{Br}$ J Radiol 1979; 52: 840-841. 
6. Daliakopoulos SI, Bairaktaris A, Papadimitriou D, Pappas P. Gigantic retroperitoneal hematoma as a complication of anticoagulation therapy with heparin in therapeutic doses: a case report. J Med Case Rep 2008; 2:162.

7. Doganay S, Kocakoc E, Balaban M. Nontraumatic hepatic hematoma caused by Wegener's granulomatosis: an unusual cause of abdominal pain. N Z Med J 2010; 123: 73-78.

8. Shankar S. Subcapsular Liver Hematoma in Metastatic GIST Complicating Imatinib (Gleevec) Therapy. Radiology Case Reports 2007; 2: $1-5$.

9. Lee BC, Ormsby EL, McGahan JP, Melendres GM, Richards JR. The utility of sonography for the triage of blunt abdominal trauma patients to exploratory laparotomy. AJR Am J Roentgenol 2007; 188: 415-421.

10. Van der Vlies CH, Olthof DC, Gaakeer M, Ponsen KJ, van Delden OM, Goslings JC. Changing patterns in diagnostic strategies and the treatment of blunt injury to solid abdominal organs. Int J Emerg Med 2011; 4: 47.

11. Ndzengue A, Hammoudeh F, Brutus P, et al. An obscure case of hepatic subcapsular hematoma. Case Rep Gastroenterol 2011; 5: 223-226.

12. Lee JW, Kim S, Kwack SW, et al. Hepatic capsular and subcapsular pathologic conditions: demonstration with CT and MR imaging. Radiographics 2008; 28: 1307-1323.

13. Lynch J, Etkind S. Spontaneous liver haematoma as a result of thrombolytic therapy. Grand Rounds 2010; 10: 38-41.

14. Swensen SJ, McLeod RA, Stephens DH. CT of extracranial hemorrhage and hematomas. AJR Am J Roentgenol 1984; 143: 907-912.

15. Ryu JK, Jin W, Kim GY. Sonographic appearances of small organizing hematomas and thrombi mimicking superficial soft tissue tumors. J Ultrasound Med 2011; 30: 1431-1436.

16. Song HP, Yu M, Zhang M, et al. Diagnosis of active hemorrhage from the liver with contrast-enhanced ultrasonography after percutaneous transhepatic angioplasty and stent placement for Budd-Chiari syndrome. J Ultrasound Med 2009; 28: 955-958.

17. Badea R, Seicean A, Procopeț B, Dina L, Osian G. Pseudoaneurysm of splenic artery ruptured in pancreatic pseudocyst and complicated by wirsungorrhagia: the role of the ultrasound techniques and contrast substances. Ultraschall Med 2011; 32: 205-207.

18. Catalano O, Aiani L, Barozzi L, et al. CEUS in abdominal trauma: multi-center study. Abdom Imaging 2009; 34: 225-234. 